\title{
sciendo
}

\section{Shorter but More Frequent Rest Periods: No Effect on Velocity and Power Compared to Traditional Sets not Performed to Failure}

\author{
by \\ Ivan Jukic ${ }^{1,2}$, James J. Tufano ${ }^{3}$
}

\begin{abstract}
Performing traditional sets to failure is fatiguing, but redistributing total rest time to create short frequent sets lessens the fatigue. Since performing traditional sets to failure is not always warranted, we compared the effects of notto-failure traditional sets and rest redistribution during free-weight back squats in twenty-six strength-trained men (28 $\pm 5.44 \mathrm{y} ; 84.6 \pm 10.5 \mathrm{~kg}, 1 \mathrm{RM}$-to-body-mass ratio of $1.82 \pm 0.33$ ). They performed three sets of ten repetitions with 4 min inter-set rest (TS) and five sets of six repetitions with 2 min inter-set rest (RR6) at 70\% of one repetition maximum. Mean velocity $(p>0.05 ; d=0.10(-0.35,0.56))$ and mean power $(p>0.05 ; d=0.19(-0.27,0.64))$ were not different between protocols, but the rating of perceived exertion (RPE) was less during RR6 $(p<0.05 ; d=0.93(0.44$, 1.40)). Also, mean velocity and power output decreased (RR6: $14.10 \%$ and 10.95\%; TS: $17.10 \%$ and 15.85\%, respectively) from the first repetition to the last, but the percentage decrease was similar (velocity: $p>0.05 ; d=0.16$ ($0.30,0.62)$; power: $p>0.05 ; d=0.22(-0.24,0.68)$ ). These data suggest that traditional sets and rest redistribution maintain velocity and power output to a similar degree when traditional sets are not performed to failure. However, rest redistribution might be advantageous as $R R 6$ displayed a lower RPE.
\end{abstract}

Key words: cluster sets, velocity, power output, rest redistribution, resistance training, training effort.

\section{Introduction}

As the importance of high power output during sport-specific movements is wellestablished, the ability to express high power output is considered to be one of the most important characteristics of an athlete (Haff and Nimphius, 2012; Suchomel et al., 2016). Therefore, power output seems to be the determining factor that differentiates between performance of athletes in a variety of sports (Bevan et al., 2010; Nimphius et al., 2010), and to increase power output, resistance training (RT) is often implemented using a variety of loads and exercises. As a result, it is important to understand the effects of different RT protocols on power output to optimize training adaptations.

Regardless of how an RT protocol is designed, maintaining movement velocity seems to be the key for maintaining power output over the course of an acute RT session, especially when the force requirements remain relatively unchanged (Oliver et al., 2016a; Tufano et al., 2016). In addition, it has been shown that decreases in movement velocity during RT are a valid indicator of neuromuscular fatigue (Sánchez-Medina and González-Badillo, 2011), which can be detrimental to power development. Furthermore, fatigue is exacerbated when performing multiple repetitions in sequence (i.e. traditional sets) (Gorostiaga et al., 2010; Moreno et al., 2014; Oliver et al., 2016b), which is why a growing body of literature is investigating the effects of different strategies to dissipate fatigue and maintain movement velocity and power output during RT.

One of the most basic yet effective methods for maintaining power output is the use

1 - Faculty of Kinesiology, University of Zagreb, Zagreb, Croatia.

2 - Athletic Lab, Sport Performance Training Center, North Carolina, USA.

3 - Faculty of Physical Education and Sport, Charles University, Prague, Czech Republic. 
of cluster sets that include short (e.g. 15-45 s) intra-set rest periods in addition to longer (e.g. $<1$ min) inter-set rest periods (Haff et al., 2003; Tufano et al., 2016, 2017a). Despite their effectiveness, it can be argued that some of the cluster set structures that have been used in research are not practical, as they can extend total training time by up to $64-169 \%$, depending on the frequency and duration of the intra-set rest intervals (Tufano et al., 2017b). Oftentimes, the time constraints of an individual training session or even of the phase of the season may be a limiting factor to consider when aiming to optimize RT sessions. Therefore, one alternative to these lengthy cluster set structures is to redistribute the total rest time of traditional set structures by abbreviating the inter-set rest, but including shorter and more frequent rest intervals.

This strategy, known as rest redistribution, has been shown to be effective in numerous studies (Joy et al., 2013; Oliver et al., 2016b; Tufano et al., 2017c) that collectively show that when total rest time is the same, shorter but more frequent rest periods are most effective for maintaining acute RT performance. However, relatively few studies have examined such set structures in high-volume free weight RT (Joy et al., 2013; Oliver et al., 2016a, 2016b), many of which included traditional sets that were performed to failure (Iglesias-Soler et al., 2012; Joy et al., 2013; Oliver et al., 2016b). In doing so, these study designs resulted in fewer repetitions, a decrease in the external load, or both during traditional sets performed to failure. Another study compared a cluster set protocol to two different rest redistribution protocols using sets of 1 and 4 repetitions, but this study did not have a traditional set protocol (Tufano et al., 2017c). Additionally, studies that examined explosive exercises like bench throws and plyometric jumps (Baker and Newton, 2007; Moreno et al., 2014) suggested that no more than five repetitions should be performed in a row in order to avoid decreases in velocity and power output and to allow for maximal recovery. However, no studies have investigated the effect of high volume restredistribution sets compared to traditional sets not performed to failure (i.e. with an equal training load and volume) in a free weight exercise.
Examining such protocols would shed light on the influence of both rest period frequency and duration on neuromuscular fatigue as well as velocity and power measures during RT when compared to traditional protocols. Since strength and conditioning professionals must often operate under time constraints, and given the importance of power development for athletic performance, it is logical to continue investigating the most efficient ways to induce power adaptations and maintain high movement velocities during training. Therefore, the purpose of our study was to compare the effects of a traditional set structure not performed to failure and a rest redistribution protocol on the velocity, power output, and perceptual responses during a high-volume free-weight back squat session where the number of repetitions and the load were both equal. Based on a study by Oliver et al. (2016b), it was hypothesized that the rest redistribution protocol would allow for greater movement velocity and power output maintenance as well as a lower fatigue perception compared to traditional sets.

\section{Methods}

\section{Participants}

Twenty-six strength-trained men (amateur weightlifters and track and field athletes) participated in this study (age $28 \pm 5.4 \mathrm{y}$, body mass $84.6 \pm 10.5 \mathrm{~kg}$ ), had at least 1 year of strength training experience using the free weight back squat exercise, and could back squat at least $100 \%$ of their body mass. Participants were excluded if they reported any recent musculoskeletal injuries. Participants averaged a 1-repetition maximum (1RM) of $152.7 \pm 25.9 \mathrm{~kg}$, resulting in a $1 \mathrm{RM}$-to-body-mass ratio of $1.82 \pm$ 0.33 .

All procedures were carried out in accordance with the Declaration of Helsinki and were approved by the University Human Research Ethics Committee. All participants gave written informed consent prior to participating.

Study Design

Testing occurred over 3 sessions: a 1RM session and 2 experimental sessions. Using a randomized counterbalanced design, participants completed each of the 2 protocols on separate days, 5-7 days apart, and were instructed to refrain from any type of fatiguing lower body 
activity for at least 48 hours before sessions. Each protocol consisted of 30 repetitions of free-weight back squats using $70 \% 1 \mathrm{RM}$, and each of the protocols consisted of a different set structure defined by different rest periods (Figure 1). Participants were asked 1 minute after the completion of each protocol for their rating of perceived exertion (RPE) scores. Previous studies have shown that a load of $70 \% 1 \mathrm{RM}$ equates to roughly a 12RM in the Smith machine back squat (Sánchez-Medina and González-Badillo, 2011). Therefore, to ensure that training to failure was avoided and that the total number of repetitions was equal between conditions, an external load of $70 \%$ was chosen. As a result, all participants successfully completed all 30 repetitions in both protocols.

\section{Repetition-Maximum Testing: Session 1}

Participants were familiarized with the protocols and anthropometrics were measured. After a dynamic warm-up (6-8 minutes), participants performed 20 barbell squats followed by 8 repetitions at $50 \%$, and 5 repetitions at $60 \%$ of their estimated $1 \mathrm{RM}$, respectively. Back squat $1 \mathrm{RM}$ was then assessed starting at $80 \%$ estimated 1RM with 2-3 minutes of rest between each successive attempt, and load was progressively increased until the 1RM was achieved using previously established methods (Matuszak et al., 2003). Participants were required to reach a depth of the squat at which the top of the thighs was at least parallel to the floor as determined by investigators for an attempt to be considered successful. During the 1RM session, participants were also familiarized with the 0-10 OMNI-RES scale: a resistance training specific RPE scale (Morishita et al., 2013).

\section{Experimental Testing: Sessions 2 and 3}

These sessions used the same warm-up as session 1, but included warm-up loads based on the actual 1RM. Each session consisted of a different, counter-balanced protocol. Specifically, the traditional set protocol (TS) consisted of 3 sets of 10 repetitions with $70 \% 1 \mathrm{RM}$ with inter-set rest intervals of $4 \mathrm{~min}$, and the rest-redistribution protocol (RR6) consisted of 5 sets of 6 repetitions at $70 \% 1 \mathrm{RM}$ with inter-set rest periods of $2 \mathrm{~min}$. The schematic view of described set structures can be seen in Figure 1.

In an attempt to maximize back squat velocity and power output, participants were instructed to perform the concentric phase of each squat as quickly as possible to a standing position (Cormie et al., 2007), while the barbell was consistently lowered under control during all repetitions in both protocols. To ensure natural squatting patterns in these experienced resistancetrained men, each participant adopted a shoulder width stance and used a self-regulated eccentric velocity; immediately upon reaching the bottom of their squat, participants were instructed to perform the concentric (upward) portion of each repetition "as explosively as possible". Verbal encouragement was provided throughout all trials. Participants were again required to reach a depth of the squat at which the top of the thighs was at least parallel to the floor as determined by the investigators for a repetition to be considered successful, however, there were no repetitions that the investigators deemed unsuccessful, indicating that the experienced participants maintained their full-squat technique throughout the entire experiment. During all repetitions, the feet were required to maintain contact with the floor (i.e. no jumping or lifting of the heels) (Cormie et al., 2007) and a slight pause was required at the conclusion of each repetition to ensure full hip and knee extension. One minute after completing each protocol, participants were asked to rate their session on a 0-10 RPE scale.

\section{Data Acquisition and Preparation}

All 30 repetitions during the back squat exercise in each of the set structures were measured with the PUSH band, which is a smartphone-based wearable device designed to track movement velocity during a variety of resistance exercises (PUSH Inc., Toronto, Canada). According to the manufacturer's guidelines, the PUSH band was worn on the participant's dominant forearm, with the hand supinated, on top of the ulna, $1-2 \mathrm{~cm}$ distal to the elbow, and with the main button located proximally. PUSH determined velocity by measuring the linear accelerations and angular velocities of the movement where vertical velocity was calculated by the integration of acceleration with respect to time. Force estimations by the PUSH were calculated from the system mass multiplied by the acceleration data, whereas power values were determined from the product of the force and velocity curve data (Balsalobre-Fernández et al., 2016). The PUSH band's sampling rate was 200 
$\mathrm{Hz}$, and to record the measured data with the PUSH band, the system was linked to an iPod (Apple, Inc., California, US) running the PUSH application v.3.1.7 using a Bluetooth 4.0 LTE connection. Afterwards, raw data were exported from the PUSH portal Internet Cloud to Microsoft Excel (Microsoft, Seattle, WA, USA) where they were prepared for later statistical analysis. The device has been proven valid and reliable in previous research (Balsalobre-Fernández et al., 2016; Sato et al., 2015).

From each repetition, mean velocity (MV) and mean power output (MP) were recorded. Similarly to previous research (Tufano et al., 2016), the percentage decline of both MV (MVD) and MP (MPD) was calculated as a percentage of the quotient of the $30^{\text {th }}$ repetition to the first repetition during RR6 and TS. Additionally, to provide a more holistic view of MV and MP throughout each protocol, the overall maintenance of MV (MVM) and MP (MPM) was calculated by dividing each repetition (1-30) by the first repetition and then averaging those values. Similarly to previous research (Cormie et al., 2009; Stone et al., 2003), we divided our participants into stronger ( $>150 \mathrm{~kg} 1 \mathrm{RM}$ squat) and weaker ( $\leq 150 \mathrm{~kg} 1 \mathrm{RM}$ squat) groups using an arbitrary value of $150 \mathrm{~kg} 1 \mathrm{RM}$ to ensure an equal number of participants in both groups. Finally, the differences in duration of the eccentric phase (ECC) of all repetitions between the protocols (TS $=1.14 \pm 0.38 \mathrm{~s} ; \mathrm{RR} 6=1.05 \pm 0.28 \mathrm{~s})$ were not present $(p>0.05 ; \mathrm{d}=0.30(-0.16,0.76)$. Therefore, the potential different duration of the ECC phase of the lift that might influence the fatigue (Wilk et al., 2018a, 2018b) was assumed to be negligible.

\section{Statistical Analysis}

All data were normally distributed as determined by the Shapiro-Wilk test of normality. Means and SDs were calculated for MV, MP, RPE, MVM, MPM, MVD, and MPD. Repeatedmeasures analysis of variance (ANOVA) was used to compare means between protocols for all variables. In addition, one-way ANOVA was used to examine the differences between stronger and weaker participants for each variable. When a significant main effect or interaction was determined, a Bonferroni post-hoc test was conducted. Cohen's $d$ effect sizes with $90 \%$ confidence intervals $(90 \% \mathrm{CI})$ were used to determine practically relevant magnitude of difference, which can be interpreted as: $\mathrm{d}<0.2$ (trivial), $\mathrm{d}=0.2-0.5$ (small), $\mathrm{d}=0.5-0.8$ (moderate), and $\mathrm{d}>0.8$ (large). All statistical analyses were performed using SPSS, version 23.0 (IBM, Chicago, USA) with an a-priori level of significance set at $p<0.05$.

\section{Results}

Mean \pm SDs for MV, MP, and RPE are presented in Figures 2 and 3. There were no differences between RR6 and TS in MV (Figure $2 \mathrm{~A} ; p>0.05 ; \mathrm{d}=0.10(-0.35,0.56)$ ), MP (Figure 2B; $p>0.05 ; \mathrm{d}=0.19(-0.27,0.64)$ ), MVD (Figure $4 ; p>$ $0.05 ; \mathrm{d}=0.16(-0.30,0.62)), \operatorname{MPD}(p>0.05 ; \mathrm{d}=0.22$ $(-0.24,0.68))$, MVM (Figure $5 ; p>0.05 ; \mathrm{d}=0.12(-$ $0.34,0.56))$, or MPM $(p>0.05 ; \mathrm{d}=0.09(-0.36$, $0.55)$ ).

The RPE was significantly lower in RR6 compared to TS (Figure 3; $p<0.05 ; \mathrm{d}=0.93$ (0.44, 1.40)). Similarly, no differences were observed when participants were divided into stronger and weaker groups during RR6 in MV $(p>0.05 ; \mathrm{d}=$ $0.37(-0.28,1.03)), \mathrm{MP}(p>0.05 ; \mathrm{d}=0.15(-0.50$, $0.80)), \operatorname{MVD}(p>0.05 ; \mathrm{d}=-0.41(-1.07,0.24))$, MPD $(p>0.05 ; \mathrm{d}=-0.46(-1.12,0.20)), \operatorname{MVM}(p>0.05 ; \mathrm{d}=$ $-0.24(-0.89,0.41))$, MPM $(p>0.05 ; \mathrm{d}=-0.28(-0.93$, $0.37))$, RPE $(p>0.05 ; \mathrm{d}=-0.59(-1.25,0.08))$ as well as during TS in MV $(p>0.05 ; \mathrm{d}=0.17(-0.48,0.82))$, MP $(p>0.05 ; \mathrm{d}=-0.08(-0.73,0.56)), \operatorname{MVD}(p>0.05$; $\mathrm{d}=-0.23(-0.88,0.42))$, MPD $(p>0.05 ; \mathrm{d}=-0.35(-$ $1.00,0.30))$, MVM $(p>0.05 ; \mathrm{d}=-0.20(-0.85,0.45))$, MPM $(p>0.05 ; \mathrm{d}=-0.37(-1.03,0.28))$, RPE $(p>$ $0.05 ; \mathrm{d}=-0.13(-0.78,0.51))$.

\section{Discussion}

The main finding of this study was that redistributing rest intervals, while performing high volume RT, did not affect fatigue-induced decrements in velocity and power measures. Despite TS having a greater RPE, the redistribution of rest periods to include more frequent but shorter sets during RR6 resulted in similar MV, MP, MVM, MPM, MVD, and MPD between the protocols. Hence, velocity and power output decreased in both protocols, but were not better maintained when more frequent inter-set rest periods were implemented with respect to the same load and total training volume while the perceptional fatigue was decreased. These results are in contrast with a large majority of studies in which higher frequency rest intervals allowed for 
greater maintenance of mean velocity and mean power output during RT (Joy et al., 2013; Oliver et al., 2016b, 2016a; Tufano et al., 2017c). There are a few potential explanations for our contradictory and novel findings.
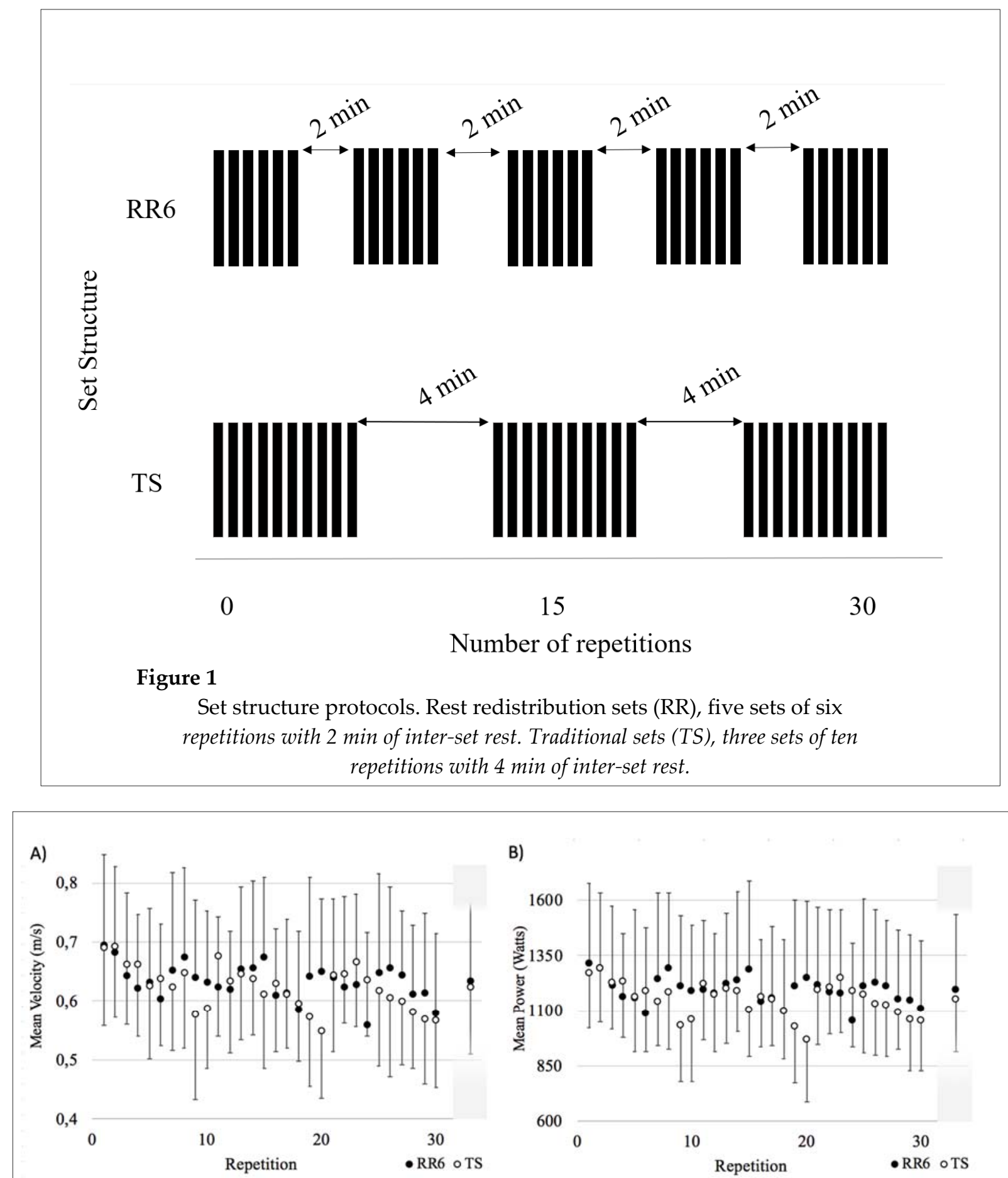

Figure 2

Means and standard deviations during rest redistribution sets (RR6) and traditional sets (TS) across 30 repetitions for: A) mean velocity output and B) mean power output. Open circles indicate velocity and power data for TS while closed circles represent velocity and power data for RR6. The shaded region on the right shows that no significant differences were present between the protocols $(p>0.05)$ when averaging all 30 repetitions together. 


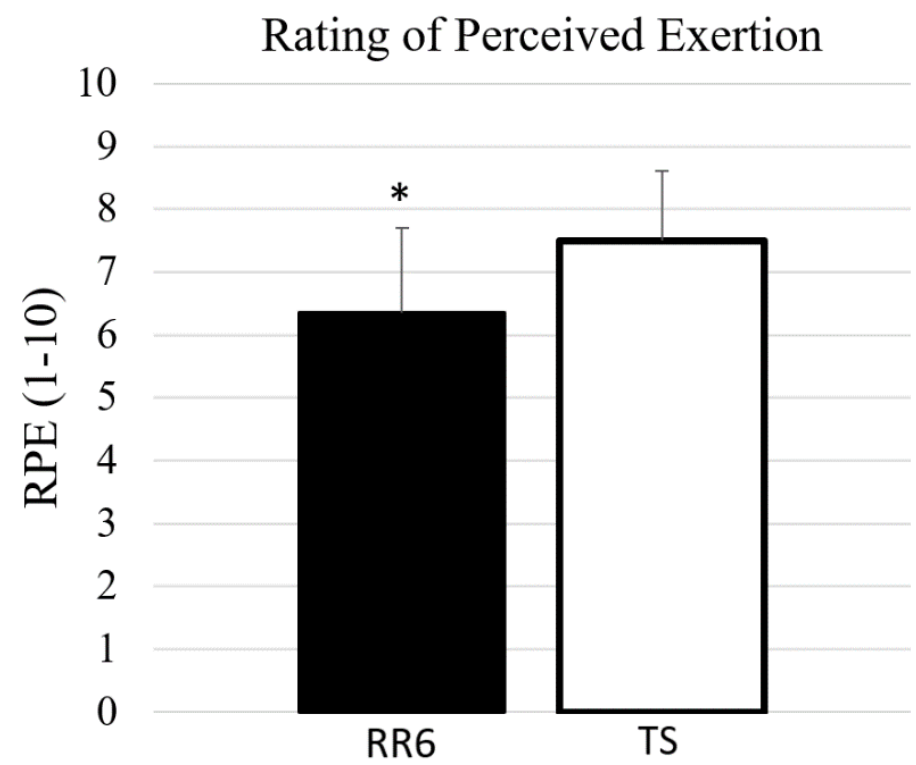

Figure 3

Means and standard deviations for the rating of perceived exertion (RPE) in both rest redistribution sets (RR6) and traditional sets (TS). Significantly less than $\operatorname{TS}^{*}(p<0.05)$

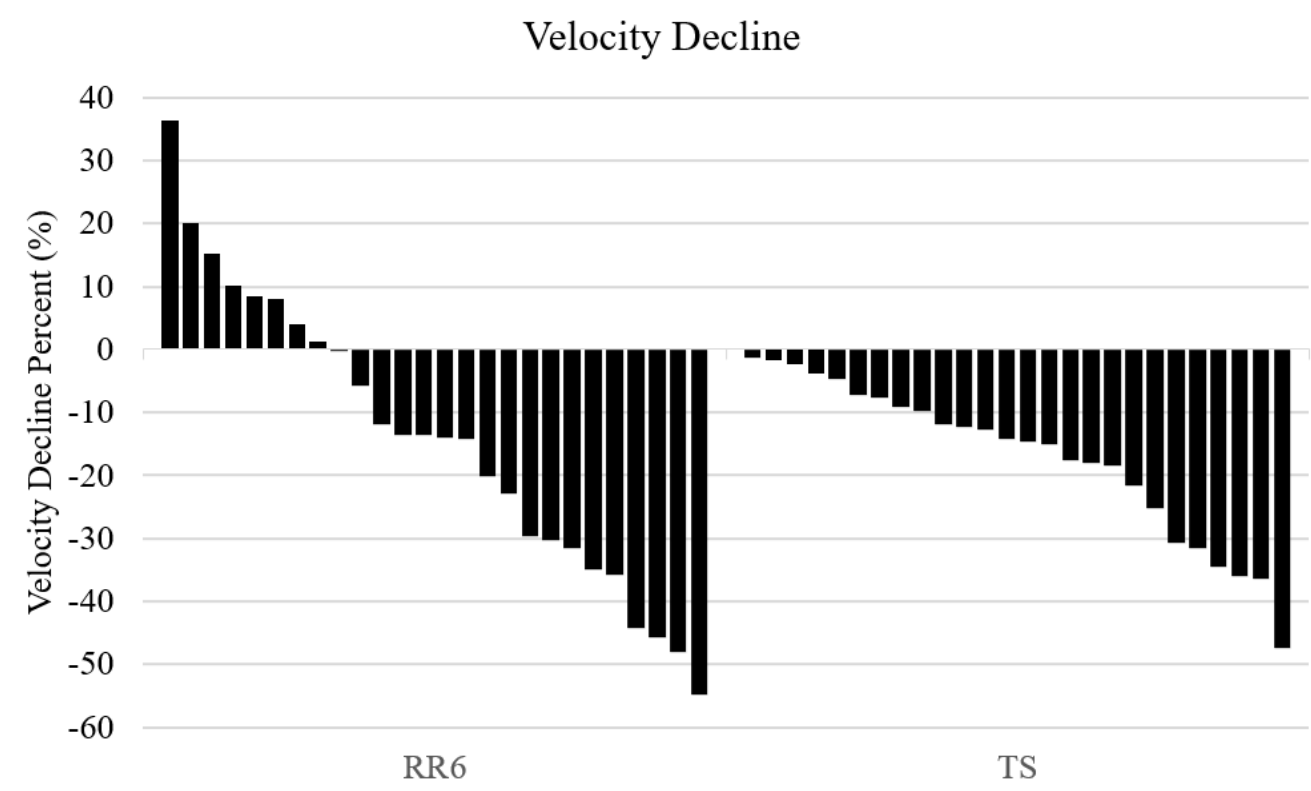

Figure 4

Individual data for mean velocity decline (MVD) expressed as a percentage of the quotient of the $30^{\text {th }}$ repetition to the $1^{\text {st }}$ repetition during RR6 and TS. Each bar represents the MVD for a single participant, the order of which is not matched or subject-dependent but is ordered according to the absolute value. For the sake of simplicity, mean power decline is not shown, as it followed the exact same pattern as $M V D$. 


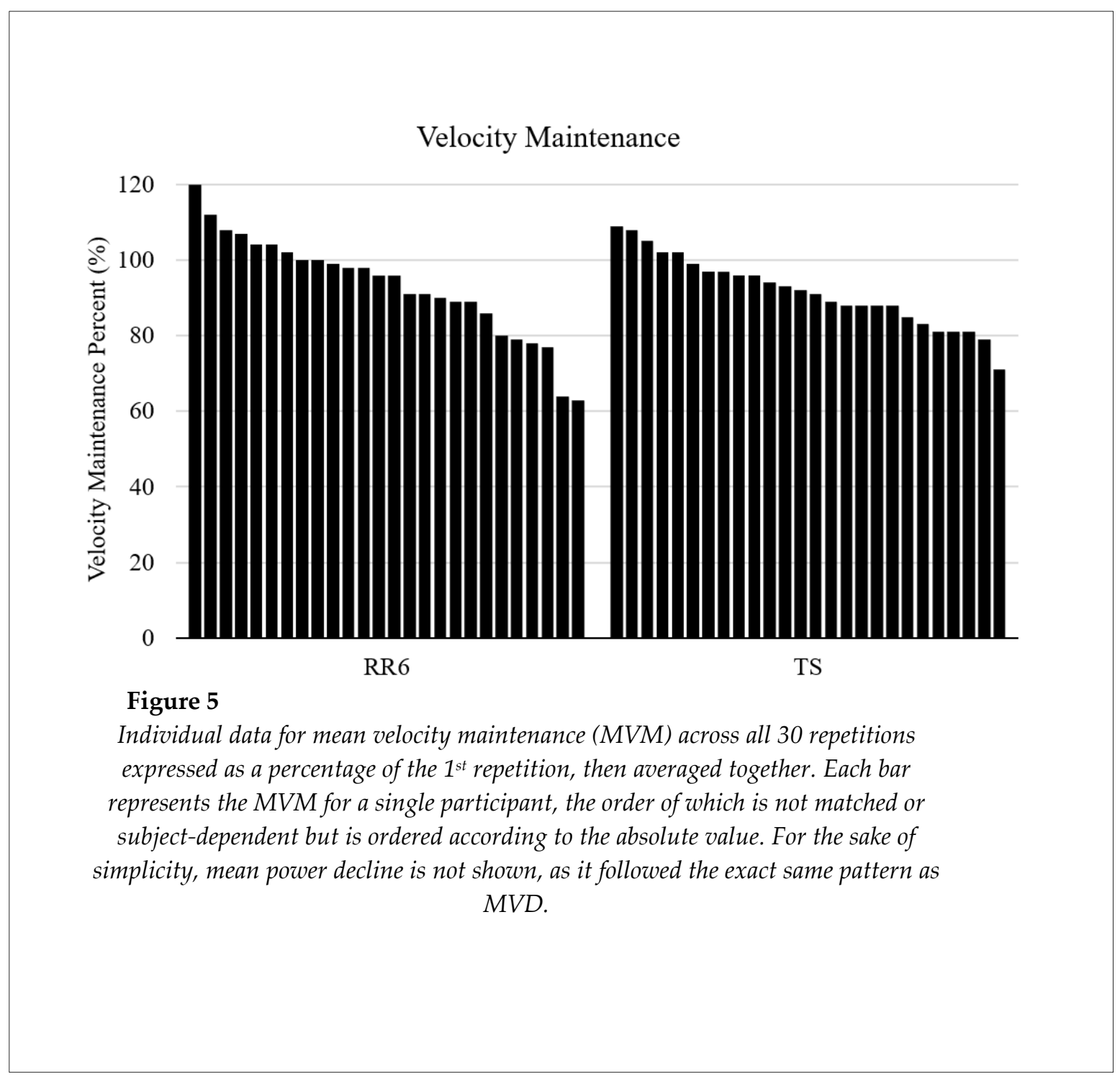

Firstly, the number of repetitions per set may have played a role. In one study (Tufano et al., 2017c), researchers compared rest redistribution protocols that consisted of either 36 one-repetition sets or 9 four-repetition sets, showing that although both protocols maintained movement velocity and power output better than a cluster set structure with longer and less frequent rest periods, the protocol with the most frequent rest periods (i.e. performing one repetition at a time) was superior for maintaining movement velocity. On the other end of the spectrum, redistributing rest periods to create six repetitions per set, which was the number of repetitions in RR6 of this study, was not effective at maintaining velocity and power output better than TS with more repetitions per set. This is in line with the findings of others (Baker and Newton, 2007; Moreno et al., 2014) who suggested that executing more than five repetitions is detrimental to power development. This is likely the case due to adenosine triphosphate (ATP) and phosphocreatine (PCr) availability reduction (Gorostiaga et al., 2010, 2012, 2014), and increased lactate and ammonia accumulation (Gorostiaga et al., 2014) that has been shown to occur when multiple consecutive repetitions are performed over multiple sets. However, the same authors suggested that increasing the frequency of rest intervals can lower lactate levels and allow for greater power output, ATP stores, and maintenance of PCr stores throughout RT. Thus, all of these factors seem to play an important role in modulating both peripheral and central fatigue 
(Zajac et al., 2015). Although such data were not measured in the present study, our data (e.g. similar MVD and MPD as well as MVM and MPM across the 30 repetitions during RR6 compared to TS) further indicate that coaches should aim for implementing a lower number of repetitions $(\leq 5)$ in the set during $\mathrm{RT}$ when the goal is to maximize power adaptations (Moreno et al., 2014). Therefore, it is likely that it may be optimal to redistribute total rest time by creating sets of up to four repetitions, but no more than six. Although the current data support this hypothesis, more research is needed to substantiate this claim.

Secondly, although one study (Mayo et al., 2016) showed that shorter but more frequent rest periods in the leg press exercise can positively impact MV when compared to traditional sets that do not lead to failure, many studies in free weight exercises that compared rest redistribution set structures to traditional sets had to either exclude some participants from the analysis (Joy et al., 2013) or decrease the load due to some subjects reaching momentary failure during traditional sets (Oliver et al., 2016a, 2016b). As participants in our study were able to complete all repetitions (3 sets of 10 repetitions with $70 \% 1 \mathrm{RM}$ ) with RPE values averaging 7.5 out of 10 (versus an assumed 10 out of 10 during high-volume training to failure in other studies), it is possible that the effects of rest redistribution on performance maintenance are not as pronounced when sets are not performed to (or nearing) concentric muscular failure. Therefore, rest redistribution is likely advantageous when performing high-volume free weight RT performed to or near concentric failure, but likely does not have as large of an effect when resistance training is not performed to failure.

The TS protocol of this study was likely not as fatiguing as the traditional set protocols of other studies because of the greater total rest time in our study. The TS protocol included $4 \mathrm{~min}$ of rest between sets, which is double than what has been used in other similar studies. Specifically, Oliver et al. (2016b) compared the effects of rest redistribution protocols that consisted of traditional sets with $2 \mathrm{~min}$ inter-set rest and another protocol with $30 \mathrm{~s}$ intra-set rest and $90 \mathrm{~s}$ of inter-set rest. As expected, their traditional set protocol was extremely fatiguing, even requiring some participants to decrease the load during testing to ensure that the prescribed number of repetitions could be completed. Similar to their organization of the set structures and findings in general, Joy et al. (2013) showed greater power output after traditional sets with 2 min inter-set rest that were divided into twice as many sets with half of the inter-set rest period (1 min instead of $2 \mathrm{~min}$ ). Although both of these studies used 70$75 \%$ of the participants' back squat $1 \mathrm{RM}$, only 2 min of rest in total were provided to execute four sets of ten repetitions with that load. This was considerably lower than the total amount of rest in the RR6 and TS protocols of the present study. Additionally, another study (Iglesias-Soler et al., 2012) also showed greater velocity and power output after a rest redistributed set configuration with a 4RM load in the parallel back squat when compared to the traditional set. Although the differences were profound between the protocols, this was likely the case due to the traditional set being intentionally performed to mechanical failure, based on which the number and length of rest periods in rest redistribution set configuration were determined (Iglesias-Soler et al., 2012). Moreover, only one repetition was performed during the rest redistribution protocol per set. Collectively, these data indicate that a lack of differences in velocity and power output of our study may have been due to the lack of extreme fatigue during TS. Therefore, it can again be concluded that redistributing rest periods to create shorter, but more frequent sets only brings a significant advantage when a comparative traditional set structure is extremely fatiguing.

Despite both the TS and RR6 protocols being seemingly similar in terms of movement velocity and power output, the RPE was significantly greater for TS compared to RR6, which suggests that it is perceptually harder to perform ten than six repetitions during multiple sets when the total rest time is equal. In addition, the RPE has been shown to simultaneously increase as movement velocity decreases (Mayo et al., 2014; Tufano et al., 2017d), which further highlights the relationship between velocity loss during RT and the degree of fatigue. However, as the maintenance of mechanical variables was similar between the protocols and the RPE was significantly different, perhaps the number of repetitions in each protocol played a role in the difference in the RPE. Studies that have 
investigated the relationship between the RPE and the number of repetitions being performed in a set support this statement ( $\mathrm{O}^{\prime}$ Connor et al., 2002; Robertson et al., 2003; Testa et al., 2012). Participants in these studies, which had similar study designs, were asked to rate their exertion levels following various numbers of repetitions during RT at a target voluntary contraction intensity, and it was found that the RPE increased with the number of repetitions at the target voluntary contraction intensity ( $\mathrm{O}^{\prime}$ Connor et al., 2002; Robertson et al., 2003; Testa et al., 2012). Therefore, it is possible that the greater number of repetitions performed in sequence during TS simply felt more difficult compared to performing more sets, but with only six repetitions per set. Furthermore, despite the fact that protocols were prescribed relative to an individual's 1RM, the stimulus of RT could differ between the protocols since individual muscular endurance also plays an important role in determining the RT stimulus (Dankel et al., 2017). Perhaps the TS protocol was perceived as more intense to the majority of the participants due to them being closer to mechanical failure than when they performed the RR6 protocol.

To further explain why the RPE may have been lower during RR6, we can delve into the comparisons between stronger (back squat $1 \mathrm{RM}>$ $150 \mathrm{~kg}$ ) and weaker participants (back squat 1RM $\leq 150 \mathrm{~kg}$ ). Of all the variables measured in this study, differences (moderate effects) were only present between stronger and weaker participants for the RPE and only during RR6, where stronger participants perceived it easier (RPE of 5.9) as opposed to their weaker counterparts (RPE of 6.7). The lack of differences between stronger and weaker participants for all other variables is in agreement with the findings from Oliver et al. (2016b) who showed similar patterns in all mechanical variables between trained and untrained individuals during both cluster and traditional set structures. However, the RPE was not measured in that study, making it difficult to attribute a strength level or training experience to the difference in the RPE during RR6. Nevertheless, both stronger and weaker participants in the present study experienced the same level of fatigue during the TS protocol which also potentially explains the fact that 8 participants in this study reacted positively to the
RR6 protocol (Figure 4).

Although these explanations for our findings are logical, this study is not without limitations. For example, when looking at the data from individual participants, it can be seen that a considerably higher proportion of them experienced lower MVD and MPD during RR6 compared to TS (Figure 4). Furthermore, Figure 5 also shows that participants responded differently to each protocol because a greater number of participants actually improved their velocity output towards the 30th repetition during RR6 which was not the case in TS where all participants experienced a decrease. Therefore, although the mean changes across all participants indicate that RR6 and TS were essentially equal, perhaps some individuals would benefit from implementing protocols similar to RR6. Additionally, due to its rising popularity, portability, and ease of use, we decided to use the PUSH device in the present study. However, it should be noted that although it has previously been shown to be valid and reliable (BalsalobreFernández et al., 2016), slight deviations compared to gold standard measurements may mask differences between protocols that might be seen using direct velocity and power measurements (e.g. force plates and linear position transducers). As such, it is up to the strength and conditioning professional to determine whether using gold standard devices is worth the cost and space, or whether more affordable and user-friendly devices such as PUSH are to be used.

Finally, based on the findings of the present study, it seems that rest redistribution protocols performed at $70 \%$ of $1 \mathrm{RM}$ are not very beneficial for maintaining movement velocity compared to traditional structures when traditional sets are not extremely fatiguing. In contrast, when traditional protocols are performed very close to mechanical failure, rest redistribution might be very beneficial, as previous studies have suggested (Joy et al., 2013; Oliver et al., 2016b, 2016a). Considering these points, cluster sets with extra intra-rest periods are still likely the best option when the aim is to maximize acute movement velocity and power output (Haff et al., 2003; Tufano et al., 2016, 2017b, 2017a), provided that time efficiency is not an issue. Future research should continue to 
investigate the most efficient ways to organize set structures during RT in order to increase training efficiency and to prevent detrimental effects of fatigue on velocity and power, which can further lead to insufficient training adaptations.

\section{Conclusions and Practical Implications}

The present study shows the inability of RR6 to maintain MV and MP to a greater extent when compared to TS while performing free weight back squats at $70 \%$ of 1 RM. Because of that, six consecutive repetitions may be too much in a single set when designing rest redistribution protocols, especially when derived from another protocol that is not extremely fatiguing. Therefore, strength and conditioning professionals should aim to implement a lower number of repetitions $(\leq 5)$ in a set when designing rest redistribution set structures to prevent velocity and power decrement during RT and ultimately maximize power adaptations in athletes.

\section{Acknowledgements:}

We wish to thank the participants for volunteering their time and energy, and Dr. Mike Young who provided his expert opinion, access to his facilities and testing equipment.

\section{References}

Baker DG, Newton RU. Change in power output across a high-repetition set of bench throws and jump squats in highly trained athletes. J Strength Cond Res, 2007; 21(4): 1007-1011. Available at https://journals.lww.com/nsca-jscr

Balsalobre-Fernández C, Kuzdub M, Poveda-Ortiz P, Campo-Vecino JD. Validity and reliability of the PUSH wearable device to measure movement velocity during back squat exercise. J Strength Cond Res, 2016; 30(7): 1968-1974. doi:10.1519/JSC.0000000000001284

Bevan HR, Bunce PJ, Owen NJ, Bennett MA, Cook CJ, Cunningham DJ, Newton RU, Kilduff LP. Optimal loading for the development of peak power output in professional rugby players. J Strength Cond Res, 2010; 24(1): 43-47. doi:https://doi.org/10.1519/JSC.0b013e3181c63c64

Cormie P, Mccaulley GO, Triplett NT, Mcbride JM. Optimal loading for maximal power output during lower-body resistance exercises. Med Sci Sports Exerc, 2007; 23(2): $103-18$. doi:10.1249/01.mss.0000246993.71599.bf

Cormie P, McGuigan MR, Newton RU. Changes in the eccentric phase contribute to improved stretchshorten cycle performance after training. Med Sci Sports Exerc. 2010 Sep;42(9):1731-44. doi: 10.1249/MSS.0b013e3181d392e8

Dankel SJ, Jessee MB, Mattocks KT, Mouser JG, Counts BR, Buckner SL, Loenneke JP. Training to Fatigue: The Answer for Standardization When Assessing Muscle Hypertrophy? Sport Med, 2017; 47(6): 10211027. Springer International Publishing. doi:10.1007/s40279-016-0633-7

Gorostiaga EM, Navarro-Amézqueta I, Calbet JAL, Hellsten Y, Cusso R, Guerrero M, Granados C, GonzálezIzal M, Ibañez J, Izquierdo M. Energy metabolism during repeated sets of leg press exercise leading to failure or not. PLoS One, 2012; 7(7): e40621. doi:10.1371/journal.pone.0040621

Gorostiaga EM, Navarro-Amézqueta I, Calbet JAL, Sánchez-Medina L, Cusso R, Guerrero M, Granados C, González-Izal M, Ibáñez J, and Izquierdo M. Blood ammonia and lactate as markers of muscle metabolites during leg press exercise. J Strength Cond Res, 2014; 28(10): $2775-85$. doi:10.1519/JSC.0000000000000496

Gorostiaga EM, Navarro-Amézqueta I, Cusso R, Hellsten Y, Calbet JAL, Guerrero M, Granados C, GonzálezIzal M, Ibáñez J, Izquierdo M. Anaerobic energy expenditure and mechanical efficiency during exhaustive leg press exercise. PLoS One, 2010; 5(10): e13486. doi:10.1371/journal.pone.0013486 
Haff GG, Nimphius S. Training principles for power. Strength Cond J, 2012; 34(6): 2-12. doi:10.1519/SSC.0b013e31826db467

Haff GG, Whitley A, Mccoy LB, O’Bryant HS, Kilgore JL, Haff EE, Pierce K, Stone MH. Effects of different set configurations on barbell velocity and displacement during a clean pull. J Strength Cond Res, 2003; 17(1): 95-103

Iglesias-Soler E, Carballeira E, Sanchez-Otero T, Mayo X, Jimenez A, and Chapman ML. Acute ef ects of distribution of rest between repetitions. Int J Sports Med, 2012; 33: 351-358. doi:10.1055/s-0031-1299699

Joy JM, Oliver JM, Mccleary SA, Lowery RP, Wilson JM. Power output and electromyography activity of the back squat exercise with cluster sets. J Sport Sci, 2013; 1: 37-45

Matuszak ME, Fry AC, Weiss LW, Ireland TR, and McKnight MM. Effect of rest interval length on repeated 1 repetition maximum back squats. J Strength Cond Res, 2003; 17(4): 634-637. doi:10.1519/15334287(2003)017<0634:EORILO>2.0.CO;2

Mayo X, Iglesias-Soler E, Carballeira-Fernández E, Fernández-Del-Olmo M. A shorter set reduces the loss of cardiac autonomic and baroreflex control after resistance exercise. Eur J Sport Sci, 2016; 16(8): 9961004. doi:10.1080/17461391.2015.1108367

Mayo X, Iglesias-Soler E, Fernández-Del-Olmo M. Effects of set configuration of resistance exercise on perceived exertion. Percept Mot Skills, 2014; 119(3): 825-837. doi:10.2466/25.29.PMS.119c30z3

Moreno SD, Brown LE, Coburn JW, Judelson DA. Effect of cluster sets on plyometric jump power. J Strength Cond Res, 2014; 28(9): 2424-2428. doi:10.1519/JSC.0000000000000585

Morishita S, Yamauchi S, Fujisawa C, Domen K. Rating of perceived exertion for quantification of the intensity of resistance exercise. Int J Phys Med Rehabil, 2013; 1(172). doi:10.4172/2329-9096.1000172

Nimphius S, Mcguigan MR, Newton RU. Relationship between strength, power, speed, and change of direction performance of female softball players. J Strength Cond Res, 2010; 24(4): 885-95. doi:10.1519/JSC.0b013e3181d4d41d

O'Connor PJ, Poudevigne MS, Pasley JD. Perceived exertion responses to novel elbow flexor eccentric action in women and men. Med Sci Sport Exerc, 2002; 34(5): 862-868. Available at http://www.acsm-msse.org

Oliver JM, Jenke SC, Mata JD, Kreutzer A, Jones MT. Acute effect of cluster and traditional set configurations on myokines associated with hypertrophy. Int J Sports Med, 2016a; 37(13): 1019-1024. doi:10.1055/s0042-115031

Oliver JM, Kreutzer A, Jenke SC, Phillips MD, Mitchell JB, Jones MT. Velocity drives greater power observed during back squat using cluster sets. J Strength Cond Res, 2016b; 30(1): $235-43$. doi:10.1519/JSC.0000000000001023

Robertson RJ, Goss FL, Rutkowski J, Lenz B, Dixon C, Timmer J, Frazee K, Dube J, Andreacci J. Concurrent validation of the OMNI perceived exertion scale for resistance exercise. Med Sci Sports Exerc, 2003; 35(2): 333-41. doi:10.1249/01.MSS.0000048831.15016.2A

Sánchez-Medina L, González-Badillo JJ. Velocity loss as an indicator of neuromuscular fatigue during resistance training. Med Sci Sports Exerc, 2011; 43(9): 1725-1734. doi:10.1249/MSS.ObO

Sato K, Beckham GK, Carroll K, Bazyler C, Sha Z, Haff, GG. Validity of wireless device measuring velocity of resistance exercises. J Trainol, 2015; 4: 15-18. doi:10.17338/trainology.4.1_15

Stone MH, O’Bryant HS, McCoy L, Coglianese R, Lehmkuhl M, Schilling B. Power and maximum strength relationships during performance of dynamic and static weighted jumps. J. Strength Cond. Res., 2003; 17(1): 140-147

Suchomel TJ, Nimphius S, Stone MH. The importance of muscular strength in athletic performance. Sport Med, 2016; 46(10): 1419. 1449. doi:10.1007/s40279-016-0486-0

Testa M, Noakes TD, Desgorces FD. Training state improves the relationship between rating of perceived exertion and relative exercise volume during resistance exercises. J Strength Cond Res, 2012; 26(11): 2990-6. doi:10.1519/JSC.0b013e31824301d1

Tufano JJ, Brown LE, Haff GG. Theoretical and practical aspects of different cluster set structures: a 
systematic review. J Strength Cond Res, 2017a; 31(3): 848-867. doi:10.1519/JSC.0000000000001581

Tufano JJ, Conlon JA, Nimphius S, Brown LE, Banyard HG, Williamson BD, Bishop LG, Hopper AJ, and Haff GG. Cluster sets: permitting greater mechanical stress without decreasing relative velocity. Int J Sports Physiol Perform, 2017b; 12: 463-469. doi:10.1123/ijspp.2015-0738

Tufano JJ, Conlon JA, Nimphius S, Brown LE, Petkovic A, Frick J, Haff GG. Effects of cluster sets and restredistribution on mechanical responses to back squats in trained men. J Hum Kinet, 2017c 58(1): 35-43. doi:10.1515/hukin-2017-0069

Tufano JJ, Conlon JA, Nimphius S, Brown LE, Seitz LB, Williamson BD, and Haff GG. Maintenance of velocity and power with cluster sets during high-volume back squats. Int J Sports Physiol Perform, 2016; 11(7): 885-892. doi:10.1123/ijspp.2015-0602

Tufano JJ, Conlon JA, Nimphius S, Oliver JM, Kreutzer A, Haff GG. Different cluster sets result in similar metabolic, endocrine, and perceptual responses in trained men. J Strength Cond Res, 2017d; Mar 13, [Epub ahead of print], doi:10.1519/JSC.0000000000001898

Wilk M, Golas A, Stastny P, Nawrocka M, Krzysztofik M, Zajac A. Does Tempo of Resistance Exercise Impact Training Volume? J Hum Kinet, 2018a; 62(1): 241-250. doi:10.2478/hukin-2018-0034

Wilk M, Stastny P, Golas A, Nawrocka M, Jelen K, Zajac A, Tufano JJ. Physiological responses to different neuromuscular movement task during eccentric bench press. Neuroendocrinol Lett, 2018b; 39(1): 26-32

Zajac A, Chalimoniuk M, Gołas A, Langfort J, Maszczyk A. Central and peripheral fatigue during resistance exercise - A critical review. J Hum Kinet, 2015; 49(1): 159-169. doi:10.1515/hukin-2015-0118

\section{Corresponding author:}

\section{Ivan Jukic}

Faculty of Kinesiology, University of Zagreb

Horvacanski Zavoj 15, 10000 Zagreb, Croatia

Phone: +385959170963

E-mail: ivan.jukic@athleticlab.com 